\title{
Efecto del tratamiento sub-cero sobre la microestructura y dureza de fundiciones blancas alto cromo
}

\author{
Effect of sub-zero treatment on the \\ microstructure and hardness of \\ high-chromium white cast iron
}

\author{
Eliseo Hernández ${ }^{1}$, Ricardo Leiva ${ }^{1,2}$, \\ Alex Escobar ${ }^{2}$, Stella Ordoñez ${ }^{1}$
}

\footnotetext{
${ }^{1}$ Departamento de Ingeniería Metalúrgica, Facultad de Ingeniería, Universidad de Santiago de Chile, Av. L. B,

O’Higgins 3363, Estación Central, Santiago, Santiago, Chile.

e-mail: eliseo.hernandez@usach.cl; stella.ordonez@usach.cl

2 Área de Investigación y Desarrollo Metalúrgico, ME Elecmetal S.A., Santiago, Santiago, Chile.

e-mail: rleiva@elecmetal.cl; aescobaru@elecmetal.cl
}

\section{RESUMEN}

En el siguiente trabajo de investigación se analizó el efecto de la temperatura de tratamiento sub-cero sobre la transformación de austenita retenida a martensita en dos fundiciones blancas de alto cromo ASTM A532 Clase II Tipo B. El tratamiento sub-cero aplicado a cada aleación, en estado de colada, fue realizado a temperaturas de $-40,-65$ y $-180{ }^{\circ} \mathrm{C}$ con un tiempo de mantenimiento de 10 minutos; transcurridos los 10 minutos cada probeta fue expuesta a temperatura ambiente. Se realizó análisis metalográfico mediante microscopia óptica, microscopía electrónica de barrido (SEM) y análisis espectroscopia de energía dispersiva (EDS) a ambas aleaciones en estado de colada, con el objetivo de caracterizar los micro-constituyentes presentes en cada aleación. La caracterización metalográfica de las probetas sometidas a tratamiento sub-cero fue realizada mediante microscopia óptica. Además, se midió la dureza Brinell de ambas aleaciones, en estado de colada y sometidas a tratamiento sub-cero.

Las aleaciones en estudio presentan diferencias importantes en su composición química, principalmente en el contenido de molibdeno y cobre, elementos determinantes en la microestructura de éstas. Los resultados obtenidos muestran un aumento en la dureza de ambas aleaciones conforme disminuye la temperaturas de tratamiento; esto, producto de un aumento en la cantidad de austenita retenida transformada a martensita a medida que se alcanza la temperatura de fin de la transformación martensítica, Mf. Se observó que un mayor contenido de molibdeno permite la obtención de una matriz austenítica metaestable de colada. Además, el efecto de este elemento en conjunto con los demás elementos de aleación fue estabilizar la austenita, obteniendo bajas cantidades de martensita al final del tratamiento sub-cero, incluso a $-180{ }^{\circ} \mathrm{C}$.

Palabras clave: fundición blanca alto cromo, tratamiento sub-cero, austenita retenida, martensita.

\section{ABSTRACT}

The effect of the sub-zero treatment temperature on the transformation of retained austenite to martensite in two high chromium white cast irons (ASTM A532 Class II Type B) was studied. The sub-zero treatment applied to each alloy in the as cast condition was performed at $-40,-65$ and $-180^{\circ} \mathrm{C}$ for 10 minutes; after the holding time was completed the samples were cooled to room temperature. The microstructure of the as cast condition was analyzed by optical microscopy (LOM) and scanning electron microscopy (SEM) and energy dispersive spectroscopy (EDS) analysis was also performed with the aim to identify the microconstituents initially present in each alloy. The metallographic characterization of the specimens subjected to sub-zero treatment was performed by optical microscopy. Furthermore, Brinell hardness was measured in the as cast and sub-zero treated samples.

The alloys studied have important differences in chemical composition, mainly in the molybdenum and cop- 
per content, which mainly determine its microstructure. The results showed an increase in the hardness of both alloys with decreasing treatment temperature; which is due to the fact that the amount of retained austenite transformed to martensite increases as the martensite finish temperature (Mf) is reached. It was also observed that a higher molybdenum content produces a metastable austenitic matrix in the as cast samples. Furthermore, the effect of Mo and other alloying elements substantially stabilizes the austenite, obtaining low amounts of martensite after the sub-zero treatment, even at $-180^{\circ} \mathrm{C}$.

Keywords: high chromium white iron, sub-zero treatment, retained austenite, martensite.

\section{INTRODUCCIÓN}

Las fundiciones blancas alto cromo (High Chromium White Iron; HCWI) son aleaciones ferrosas que contienen entre 2,0 y $3,2 \%$ de carbono y 11,0 a 30,0\% de cromo como elemento de aleación principal [1]. Estas fundiciones presentan excelente resistencia a la abrasión, adecuada estabilidad en ambientes agresivos y una importante tenacidad, considerando su alta dureza [2,3]. Gracias a estas características, estas aleaciones son utilizadas en la fabricación de diferentes componentes como bombas de pulpa, revestimientos de molinos de bolas, bolas de molienda, equipamientos para movimiento de tierra y otras aplicaciones similares, donde el desgate y los repetidos impactos son las principales causas de falla en los materiales $[3,4]$.

Una característica de las fundiciones blancas alto cromo es su estructura en estado de colada. Por lo general, durante la solidificación la austenita formada (la cual puede ser primaria y/o eutéctica según la composición de la aleación) se encuentra saturada en carbono y cromo; esto, en conjunto con el efecto de los demás elementos de aleación, permite obtener una estructura compuesta por una matriz austenítica estabilizada a temperatura ambiente y colonias eutécticas conformadas por austenita y carburos eutécticos tipo $\mathrm{M}_{7} \mathrm{C}_{3}$ [3, $5,6,7]$. Sin embargo, variaciones en la composición química, en conjunto con las condiciones de enfriamiento (por lo general asociado a espesores de pieza), permiten la obtención de matrices ferríticas, martensíticas, bainíticas, perlíticas, incluso una mezcla de todas éstas, ampliando el rango de uso de estas aleaciones en estado de colada $[3,8]$.

El contenido de austenita retenida presente en piezas en estado de colada y tratadas térmicamente es una de las principales causas de agrietamiento superficial producto de la trasformación a martensita que ésta sufre en servicio $[4,9,10,11]$. Por este motivo y con el fin de reducir los niveles de austenita retenida presente, estas aleaciones pueden ser sometidas a diversos tratamientos térmicos [12,13]. Principalmente estas aleaciones son sometidas a tratamiento térmico de desestabilización de austenita $[3,7,14,15]$, pero también dependiendo de su composición química y estructura en estado de colada, pueden ser tratadas mediante tratamientos sub-cero [16, 17, 18]. En fundiciones blancas que presentan elevados contenidos de austenita retenida en estado de colada es posible la formación de una matriz martensítica a partir de tratamientos sub-cero, obteniendo así una mayor dureza, resistencia a la abrasión y estabilidad dimensional. Además, es posible reducir los niveles de austenita retenida en componentes tratados térmicamente mediante la aplicación de tratamientos sub-cero [3, 5, 19].

Algunas fundiciones y aceros de alta aleación presentan una temperatura de inicio de la transformación martensítica (Ms) cercana a temperatura ambiente, obteniendo considerables cantidades de austenita retenida post tratamiento térmico $[2,16,20,21]$. La reducción de la cantidad de austenita retenida, con la consecuente formación de martensita mediante la aplicación de tratamientos sub-cero se produce a medida que se alcanza la temperatura de fin de la transformación martensítica (Mf) [22,23]. En base a esta temperatura se definirá la temperatura de tratamiento sub-cero apropiada para obtener la menor cantidad de austenita retenida post tratamiento [16, 24, 25].

La composición química de la austenita presente determinará el grado de estabilidad de ésta. Por este motivo algunas fundiciones blancas son fabricadas con químicas definidas para favorecer la templabilidad de la aleación, pero sin producir una reducción considerable de la Mf, permitiendo así la obtención de una matriz martensítica posterior al tratamiento térmico sub-cero, método de producción útil en la fabricación de componentes de geometrías complejas, los cuales que pueden ser afectados durante la realización de tratamientos térmicos a alta temperatura [8].

\section{MATERIALES Y MÉTODOS}

En la siguiente investigación fueron utilizadas dos fundiciones blancas alto cromo obtenidas desde cuerpos de prueba en estado de colada de distinta aleación, facilitados por la empresa ME Elecmetal. Las dos fundiciones, de distinta composición química, fueron designadas como aleación A y aleación B. 
La aleación A fue fabricada en un cuerpo de prueba tipo Cuña; la aleación B fue fabricada en un cuerpo de prueba tipo Bloque de dos espesores. La Figura 1 presenta las dimensiones aproximadas de los cuerpos de prueba utilizados.
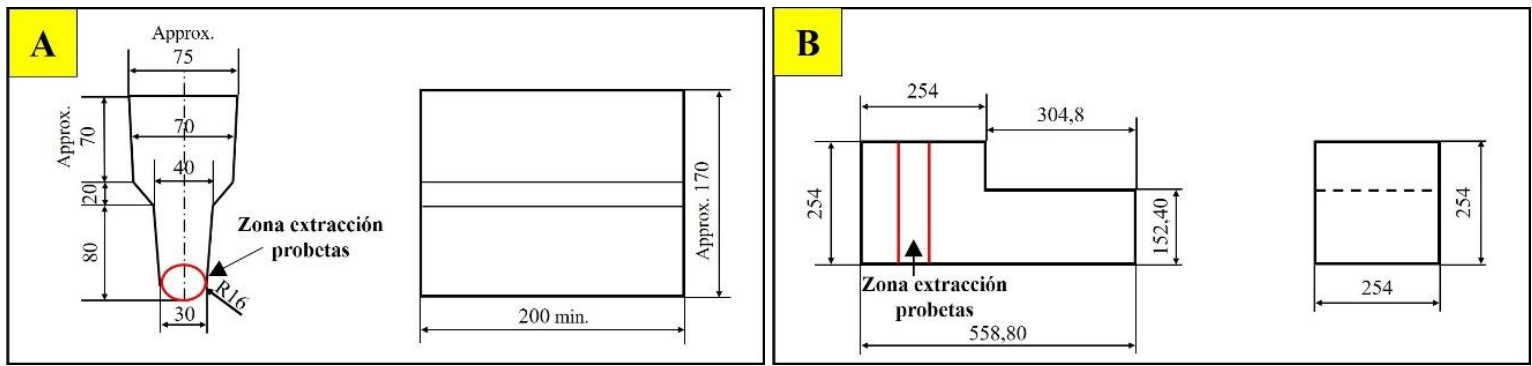

Figura 1: Dimensiones (en mm) cuerpos de prueba utilizados, (A) tipo Cuña; (B) tipo Bloque.

Desde ambos cuerpos de prueba se extrajeron secciones mediante corte por electroerosión. A partir de éstas, mediante el uso de una cortadora de banco equipada con disco de corte y sistema de refrigeración, se fabricaron 4 probetas de cada aleación, con dimensiones 10x20x20 (mm), aproximadamente.

Con el objetivo de conocer la composición química de cada aleación, se realizó análisis químico a ambas muestras en estado de colada mediante espectrometría de emisión óptica, en las dependencias de ME Elecmetal.

Los tratamientos sub-cero fueron realizados dentro de un recipiente metálico que contenía la solución refrigerante, cuya temperatura fue medida con termocuplas tipo K. Para la realización del tratamiento se utilizaron tres probetas de cada aleación en estado de colada, las que fueron sometidas a temperaturas de -40 , -65 y $-180{ }^{\circ} \mathrm{C}$. Para los tratamientos realizados a temperaturas de -40 y $-65{ }^{\circ} \mathrm{C}$, se preparó una solución refrigerante a partir de hielo seco y alcohol, adicionados en las proporciones necesarias. Primero, se introdujeron 200 gramos de hielo seco dentro del recipiente metálico, posteriormente se adicionó alcohol hasta alcanzar la temperatura deseada. Para realizar el tratamiento a $-180{ }^{\circ} \mathrm{C}$ se utilizó nitrógeno líquido como medio refrigerante. Una vez lograda la temperatura de tratamiento, se sumergió dentro del recipiente una probeta de cada aleación durante 10 minutos. Completado el tiempo de mantenimiento, las probetas fueron retiradas y expuestas a condiciones ambiente.

La preparación de muestras para metalografía fue realizada en una pulidora automática marca Struers, modelo LaboPol-5. Con el fin de identificar los micro-constituyentes presentes, las probetas fueron atacadas con dos reactivos diferentes: Vilella y LePera Modificado (Picral 4\% más Metabisulfito de sodio 10\%). Para este último ataque, se sobre ataca la muestra con Picral durante 10 a 20 segundos, luego se lava, se seca y se ataca con Metabisulfito de sodio al $10 \%$ durante 10 segundos, finalmente se lava y seca nuevamente. Para caracterización microestructural de ambas aleaciones en estado de colada se utilizó un equipo marca JEOL modelo JSM - 6010LA con el que se realizó análisis SEM mediante la técnica de electrones retrodispersados y análisis de espectroscopia de energía dispersiva (EDS) para determinar la composición química de las fases presentes en ambas fundiciones; las probetas sometidas a tratamiento sub-cero fueron caracterizadas mediante microscopia óptica (LOM).

La cuantificación de fases presentes en cada aleación en estado de colada fue realizada con el software Image-Pro Plus 6, mediante el análisis de imagen de una serie de metalografías obtenidas por microscopia electrónica de barrido y óptica a 50 y 100 aumentos. La cuantificación se realizó a partir del contraste obtenido en las fases presentes producto de los ataques metalográficos utilizados en el caso de las micrografías ópticas y de la diferencia composicional en el caso de las micrografías electrónicas. La fracción volumétrica de cada fase presente en las micrografías fue estimada en relación al área de cada una de éstas sobre el área total de la imagen.

La dureza de las muestras se midió con un durómetro de banco marca Zwick-Indentec equipado con un indentador de bola de carburo de tungsteno (WC) de $10 \mathrm{~mm}$ de diámetro. El ensayo fue realizado con carga de $3000(\mathrm{~kg})$ aplicada durante 30 segundos. 


\section{RESULTADOS}

\subsection{Análisis de composición química}

La Tabla 1 presenta los resultados del análisis químico, obtenidos mediante espectrometría de emisión óptica, de las fundiciones blancas en estado de colada, en muestras obtenidas desde cuerpos de prueba.

Tabla 1: Resultados de análisis químico. Composición química (\% peso) de fundiciones de estudio.

\begin{tabular}{c|c|c|c|c|c|c}
\hline \multirow{2}{*}{ Aleación A } & \%C & \%Cr & \%Mo & \%Cu & $\% \mathbf{M n}$ & $\% \mathbf{S i}$ \\
\cline { 2 - 7 } & 2,60 & 15,20 & 2,50 & 0,10 & 0,70 & 0,44 \\
\hline \multirow{2}{*}{ Aleación B } & $\% \mathbf{C}$ & $\% \mathbf{C r}$ & $\% \mathbf{M o}$ & $\% \mathbf{C u}$ & $\% \mathbf{M n}$ & $\% \mathbf{S i}$ \\
\cline { 2 - 7 } & 2,80 & 18,90 & 1,10 & 0,90 & 0,90 & 0,55 \\
\hline
\end{tabular}

\subsection{Caracterización de fundiciones blancas en estado de colada}

La Figura 2 muestra la microestructura de ambas aleaciones en estado de colada, obtenidas mediante microscopia electrónica de barrido. La aleación A (Figura 2 (A, B)) presenta matriz austenítica metaestable, carburos eutécticos tipo $\mathrm{M}_{7} \mathrm{C}_{3}$ y $\mathrm{Mo}_{2} \mathrm{C}$ (estos últimos de color blanco intenso) en regiones interdendríticas, junto a pequeñas cantidades de colonias perlíticas, martensita y bainita en torno a los carburos eutécticos. La aleación B (Figura 2 (C, D)) presenta una matriz formada por austenita retenida, colonias perlíticas y bainita, ubicadas mayoritariamente en el seno de la estructura dendrítica, y colonias de carburos eutécticos tipo $\mathrm{M}_{7} \mathrm{C}_{3}$ en regiones interdendríticas. Se observan mayores cantidades de martensita en la periferia de los carburos eutécticos.
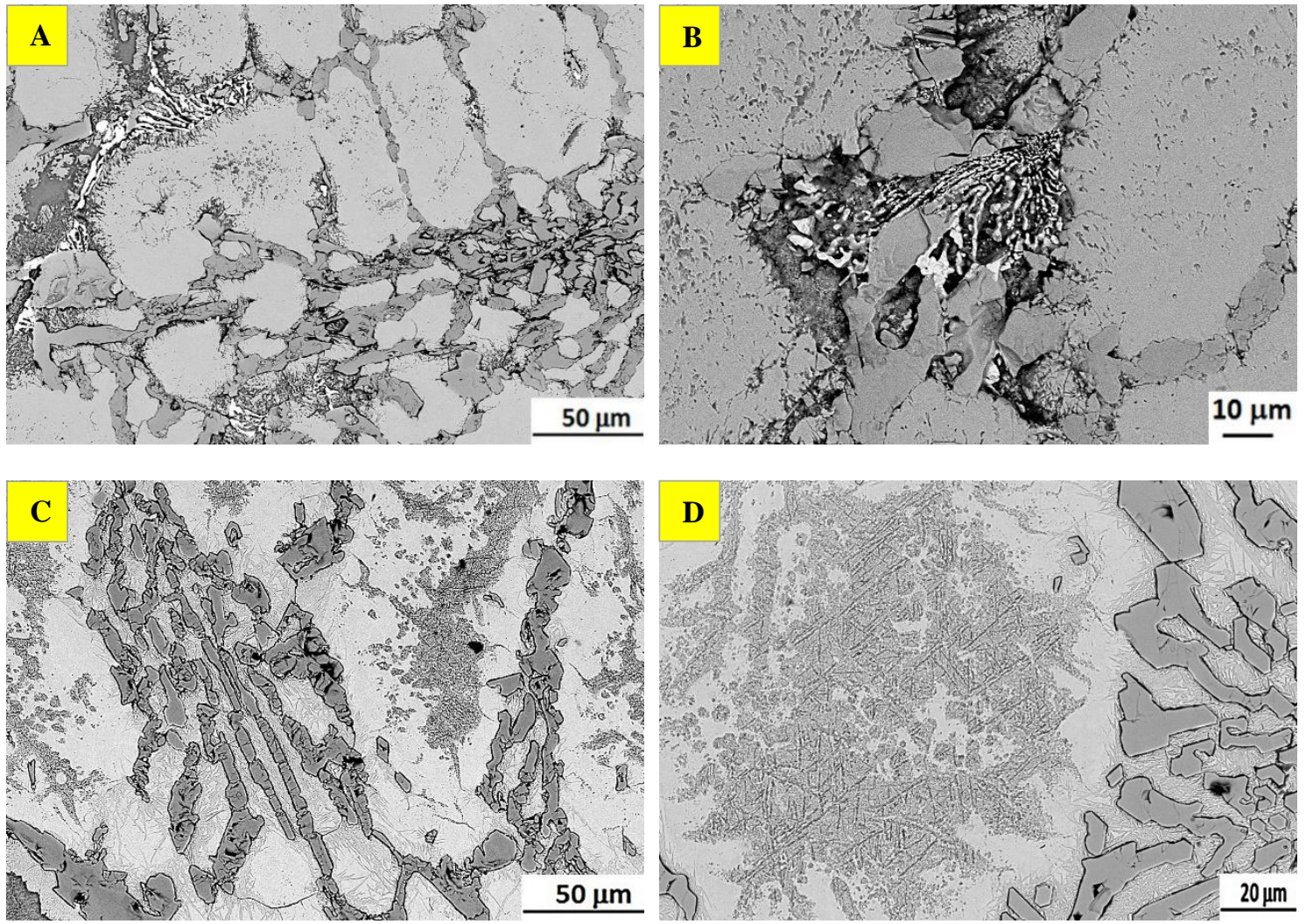

Figura 2: Micrografías electrónicas de barrido de ambas aleaciones de estudio en estado de colada. Aleación A (A, B) muestra la presencia de carburos eutécticos tipo $\mathrm{M}_{7} \mathrm{C}_{3}$ y $\mathrm{Mo}_{2} \mathrm{C}$ en una matriz autenítica; Aleación $\mathrm{B}(\mathrm{C}, \mathrm{D})$ se aprecia la presencia de carburos eutécticos tipo $\mathrm{M}_{7} \mathrm{C}_{3}$ una matriz formada por austenita retenida, colonias perlíticas y bainita. (Atacadas con Vilella). 
Como muestra la Figura 3, donde se presenta una ampliación de la micrografía de la figura 2.A, se identificó la presencia carburos eutécticos de molibdeno en la aleación A, los cuales presentan elevados contenidos de molibdeno (70 \%, aproximadamente), lo que es representado como un pico de gran intensidad en el análisis EDS respectivo, con menores cantidades de hierro y cromo.
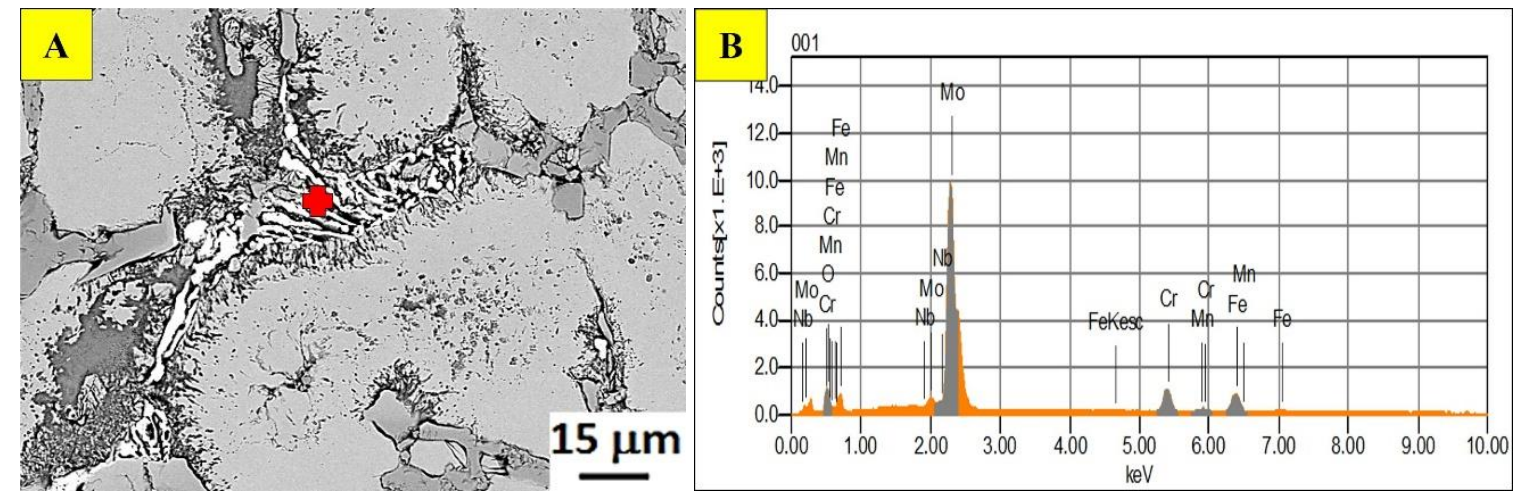

Figura 3: (A) Micrografía electrónica de barrido aleación A en estado de colada. (B) Análisis EDS del carburo rico en molibdeno, $\mathrm{Mo}_{2} \mathrm{C}$, indicado en (A). (Atacada con Vilella).

La cuantificación de microconstituyentes presentes en cada aleación en estado de colada, determinada a partir de la fracción volumétrica estimada mediante análisis de imagen por contraste de color de microconstituyentes en metalografías obtenidas por microscopia electrónica de barrido y óptica, es presentada en la Tabla 2.

Tabla 2: Cuantificación de microconstituyentes en las aleaciones A y B en estado de colada (\%vol.).

\begin{tabular}{c|c|c}
\hline Microconstituyente & Aleación A & Aleación B \\
\hline Perlita más bainita & 6,88 & 24,46 \\
\hline Martensita & 5,34 & 14,97 \\
\hline Austenita retenida & 61,13 & 33,29 \\
\hline Carburos Eutécticos & 26,65 & 28,28 \\
\hline
\end{tabular}

Con el objeto de analizar el efecto de la composición química de cada aleación en la respuesta al tratamiento sub-cero, la Tabla 3 presenta el resultado del análisis EDS realizado en regiones austeníticas de ambas aleaciones en estado de colada.

Tabla 3: Resultados del análisis EDS que muestra la composición química de la austenita metaestable presente en la matriz de la estructura de colada de ambas aleaciones, porcentaje en peso (\%peso).

\begin{tabular}{c|c|c|c|c|c}
\hline Fundición & $\% \mathbf{C r}$ & $\% \mathbf{M o}$ & $\% \mathbf{C u}$ & $\% \mathbf{M n}$ & $\% \mathbf{S i}$ \\
\hline Aleación A & 10,580 & 1,412 & 0,287 & 0,938 & 0,696 \\
\hline Aleación B & 10,900 & 0,640 & 1,240 & 1,016 & 0,793 \\
\hline
\end{tabular}

\subsection{Análisis metalográfico posterior a tratamiento sub-cero}

La Figura 4 presenta la microestructura de las aleaciones A y B en estado de colada y sometidas a tratamiento sub-cero. La aleación A sometida a tratamiento sub-cero no muestra una diferencia significativa respecto a la estructura de colada. La aleación B, en cambio, presenta un aumento gradual del contenido de martensita (revelada selectivamente en una tonalidad azul) conforme la temperatura de refrigeración es reducida. 

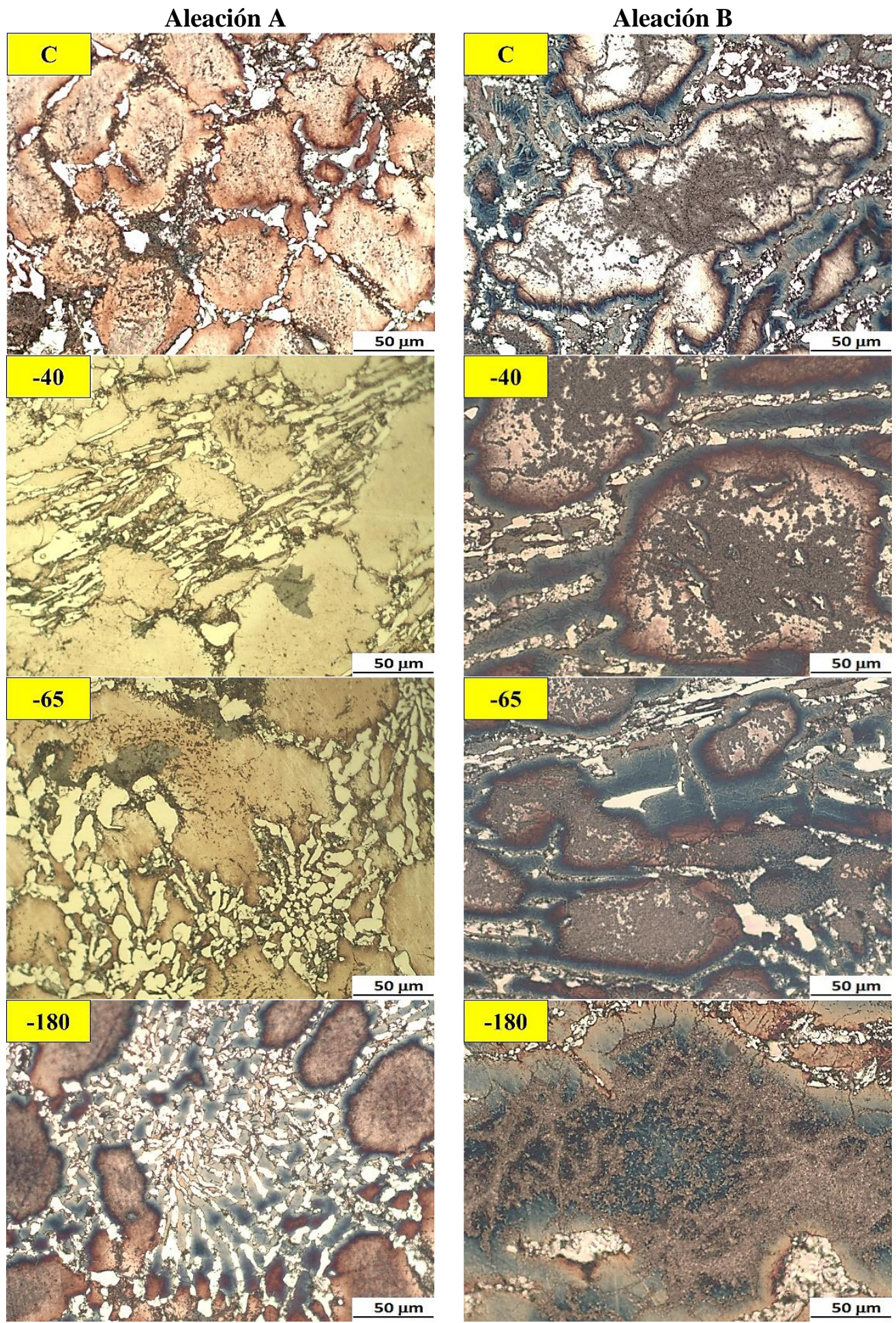

Figura 4: Metalografías ópticas obtenidas para ambas aleaciones de estudio en estado de colada y sometidas a diferentes temperaturas de sub-enfriamiento. C: Estado de colada; -40, -65 y -180: Temperaturas de tratamiento sub-cero en grados Celsius $\left({ }^{\circ} \mathrm{C}\right)$. (Ataque con LePera Modificado, excepto aleación A -40 y $-65^{\circ} \mathrm{C}$ con Vilella).

\subsection{Evaluación de dureza}

La Tabla 4 presenta el resultado de la medición de dureza Brinell realizado a cada aleación, en estado de colada y sometida a tratamiento sub-cero. Además, se incluye la diferencia de dureza obtenida para cada temperatura de subenfriamiento respecto a la estructura de colada, expresada como $\Delta \mathrm{HB}$. 
Tabla 4: Resultados de medición de dureza Brinell, para ambas aleaciones en estado de colada y con tratamientos subcero a $-40,-65$ y $-180^{\circ} \mathrm{C}$.

\begin{tabular}{|c|c|c|c|c|c|}
\hline \multicolumn{3}{|c|}{ Aleación A } & \multicolumn{3}{|c|}{ Aleación B } \\
\hline Condición & Dureza, HB & $\Delta \mathrm{HB}_{\mathrm{A}}$ & Condición & Dureza, HB & $\Delta \mathrm{HB}_{\mathrm{B}}$ \\
\hline Colada & 423 & - & Colada & 530 & - \\
\hline$-40 \stackrel{\circ}{C}$ & 429 & 6 & $-40 \stackrel{\circ}{C}$ & 564 & 34 \\
\hline 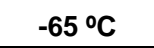 & 451 & 28 & 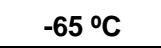 & 601 & 71 \\
\hline$-180 \stackrel{\circ}{ } \mathrm{C}$ & 477 & 54 & $-180 \stackrel{\circ}{C}$ & 611 & 81 \\
\hline
\end{tabular}

\section{DISCUSIÓN}

A partir del resultado del análisis químico, presentado en la Tabla 1, ambas fundiciones son clasificadas como Clase II Tipo B según la norma ASTM A532 [1]. Sin embargo, diferencias en la composición química de éstas generan variaciones en la microestructura y dureza de la fundición en estado de colada.

La templabilidad de la fundición estará determinada principalmente por su composición química, siendo influenciada mayoritariamente por el contenido de molibdeno, manganeso, níquel y cobre [3,8]. La templabilidad de la aleación A está atribuida principalmente al contenido de molibdeno, siendo favorecida por adiciones de manganeso. La aleación B, en cambio, pese a tener un menor contenido de molibdeno, su templabilidad es favorecida por la combinación de este elemento con una mayor cantidad de cobre y manganeso. Si bien el cobre actúa como un perlitizante, este efecto es anulado en fundiciones blancas altamente aleadas. La combinación de cobre y molibdeno $(0,5$ a 2,0\% de Mo) favorece considerablemente la templabilidad de las fundiciones blancas alto cromo, suprimiendo de forma efectiva la formación de perlita y bainita en piezas tratadas térmicamente [8].

La diferencia microestructural observada en la matriz de ambas aleaciones en estado de colada (Figura 2 y Tabla 2), en especial la cantidad de austenita retenida presente, se atribuye principalmente al contenido de molibdeno. Las aleaciones A y B contienen un 2,50\% y 1,10\% de molibdeno, respectivamente. Un aumento en el contenido de este elemento favorece notoriamente la templabilidad de las aleaciones, expresado como un mayor diámetro crítico de temple, evitando la formación de perlita y bainita post tratamiento térmico. Con el suficiente contenido de molibdeno en conjunto con manganeso, níquel y cobre, se produce un efecto similar durante el enfriamiento de las aleaciones de colada, incluso en piezas de espesores considerables, inhibiendo la formación de perlita y/o bainita durante el enfriamiento [2,26]. El mayor contenido de molibdeno en la aleación A generó, posterior al enfriamiento desde colada, la formación de una matriz compuesta mayoritariamente por austenita metaestable pese a contener menores cantidades de manganeso y cobre que la aleación $\mathrm{B}$.

Además, la presencia de carburos tipo $\mathrm{Mo}_{2} \mathrm{C}$ en la aleación A, identificados en la Figura 3, también está atribuida al mayor contenido de molibdeno de esta aleación. Producto de la presencia de carbono se favorece la formación de carburos eutécticos de molibdeno en la etapa final de la solidificación. IKEDA et al. [27], estudiaron el efecto de la adición de este elemento en la estructura de colada de fundiciones blancas alto cromo hipoeutécticas. La solidificación de la fundición comienza con la nucleación de dendritas de austenita primaria, seguida por la formación de colonias eutécticas de austenita más carburos eutécticos tipo $\mathrm{M}_{7} \mathrm{C}_{3}$ y finalmente la formación de carburos eutécticos tipo $\mathrm{Mo}_{2} \mathrm{C}$. En el estudio de Ikeda la fundición blanca aleada con un $0,93 \%$ de molibdeno no presenta el pico de la reacción exotérmica correspondiente a la cristalización del carburo $\mathrm{Mo}_{2} \mathrm{C}$ durante la solidificación. Esto concuerda con los resultados obtenidos; el menor contenido de molibdeno de la aleación $\mathrm{B}(1,10 \% \mathrm{Mo})$, no permite la cristalización del carburo tipo $\mathrm{Mo}_{2} \mathrm{C}$ durante su solidificación, a diferencia de lo que ocurre en la aleación A (2,50\%Mo), en la que si se aprecia este carburo.

La existencia de martensita en torno a la interfase matriz/carburo eutéctico, se debe a la reducción en el contenido de cromo y carbono en la austenita producto de la formación de carburos durante la solidificación de la aleación y posterior enfriamiento hasta temperatura ambiente. La reducción de estos elementos eleva la temperatura de inicio de la trasformación martensítica, favoreciendo la formación de martensita alrededor y en el interior de las colonias eutécticas [5,28].

De acuerdo a las metalografías obtenidas como resultado del tratamiento sub-cero realizado, presentadas en la Figura 4, la microestructura de la aleación A sometida a tratamiento sub-cero no presentó diferencias notorias respecto a la microestructura de colada, manteniendo una matriz de austenita metaestable inclu- 
so después de ser expuesta a $-180{ }^{\circ} \mathrm{C}$. Es posible apreciar martensita en la interfase matriz/carburos eutécticos, región empobrecida en carbono y cromo como fue discutido anteriormente. En la aleación B, en cambio, si es posible apreciar el efecto de la temperatura de tratamiento sub-cero en la microestructura de la fundición. La probeta tratada a $-40{ }^{\circ} \mathrm{C}$ no presenta mayores diferencias respecto a la estructura de colada. Con una temperatura de $-65^{\circ} \mathrm{C}$ fue posible apreciar un aumento en la cantidad de martensita en torno a las colonias eutécticas. Finalmente, a $-180{ }^{\circ} \mathrm{C}$ se obtuvo mayores cantidades de martensita, presente incluso en el seno de la matriz, asociada a regiones bainíticas. Cuando la matriz de la aleación presenta una elevada cantidad de austenita retenida, está será más resistente a desarrollar la transformación a martensita durante el tratamiento sub-cero. MARATRAY y POULALION [29] mostraron que el efecto de la respuesta al tratamiento sub-cero está asociado a la estabilidad de la austenita. En el estudio se demostró que en medida que se tiene una menor Ms, mayor es la cantidad de austenita retenida obtenida post tratamiento sub-cero, fenómeno apreciable en las aleaciones A y B.

Es importante considerar el efecto de los elementos aleantes en la estabilidad de la austenita y en la reducción de la temperatura de inicio y término de la transformación martensítica. Como muestra la Tabla 3, la austenita metaestable presente en la aleación A presenta un mayor contenido de molibdeno que la obtenida en la aleación B. Un mayor contenido de éste elemento, asociado a los demás elementos de aleación, favorece la estabilización de la austenita retenida, incluso a $-180^{\circ} \mathrm{C}$. Debido a la estabilización de la austenita (producto del enriquecimiento de ésta en elementos aleantes) presente en la aleación A no es posible la formación de cantidades considerables de martensita en el periodo de exposición a temperatura sub-cero; efecto contrario al apreciado en la aleación $\mathrm{B}$, la que presenta una matriz con bajas cantidades de austenita retenida a -180 ${ }^{\circ} \mathrm{C}$. Además, el mayor contenido de silicio en la aleación B podría favorecer la formación de mayores cantidades de martensita a partir de austenita retenida. Algunas fundiciones blancas alto cromo aleadas con considerables cantidades de silicio, que presentan una matriz compuesta por martensita y austenita en estado de colada, son sometidas a tratamiento sub-cero para obtener una matriz completamente martensítica [3]. El silicio disminuye la solubilidad de carbono en la austenita, reduciendo la templabilidad de la aleación, pero produciendo un aumento de la Ms y Mf [3, 26], favoreciendo la formación de una mayor cantidad de martensita posterior al tratamiento sub-cero. El efecto contrario del silicio en la templabilidad de la aleación se ve contrarrestado con la adición de elementos aleantes como molibdeno, manganeso, cobre y níquel $[3,8]$.

Con respecto a los resultados de dureza presentados en la Tabla 4, la aleación A presenta una matriz mayoritariamente formada por austenita retenida, en cambio, la matriz de la aleación B está constituida por perlita, bainita, austenita retenida y martensita, por lo que su dureza Brinell fue mayor. La dureza de ambas aleaciones aumentó en relación a la estructura de colada a medida que disminuyó la temperatura de tratamiento. Este efecto se produce por el aumento en la fracción de martensita, formada a partir de austenita metaestable conforme se alcanza la temperatura de fin de la transformación martensítica, Mf [20, 21, 22, 24]. La aleación A no presenta un aumento de dureza considerable al ser expuesta a $-40{ }^{\circ} \mathrm{C}$, lo que indicaría que la temperatura de inicio de la transformación martensítica para esta aleación es cercana (pero superior) a la temperatura ambiente, ya que la estructura en estado de colada presenta un 5\% de martensita, aproximadamente. A menores temperaturas de exposición, $-65{ }^{\circ} \mathrm{C}$ y $-180{ }^{\circ} \mathrm{C}$, la dureza aumentó a 451 y $477 \mathrm{HB}$, respectivamente. La aleación $\mathrm{B}$, que presentó menores cantidades de austenita retenida en estado de colada, aumentó su dureza en $34 \mathrm{HB}$ al ser expuesta a $-40^{\circ} \mathrm{C}$. Con temperaturas de tratamiento de $-65{ }^{\circ} \mathrm{C} \mathrm{y}-180{ }^{\circ} \mathrm{C}$ la dureza aumentó a 601 y $611 \mathrm{HB}$, respectivamente.

A partir de estos resultados, junto a la caracterización metalográfica de la estructura de colada, se puede determinar que la temperatura de inicio de la trasformación martensítica, en ambas aleaciones fue alcanzada sobre los $25^{\circ} \mathrm{C}$, siendo mayor en la aleación B, asociada a un mayor contenido de martensita. Además, el leve aumento de dureza obtenido en la aleación B entre $-65^{\circ} \mathrm{C}$ y $-180{ }^{\circ} \mathrm{C}$ podría indicar que la temperatura de fin de la transformación martensítica fue alcanzada en este rango de temperaturas. Por su parte, en la aleación A, que pese a presentar un aumento de dureza conforme disminuyó la temperatura de tratamiento (asociado a un aumento en el contenido de martensita), es probable que no se haya alcanzado la Mf producto del bajo nivel de dureza obtenido a $-180{ }^{\circ} \mathrm{C}$ considerando la cantidad de austenita retenida disponible para transformar a martensita. De hecho, en fundiciones blancas de matriz austenítica metaestable en estado de colada y sometidas a tratamientos sub-cero en procesos industriales, y en las que la transformación de austenita a martensita es completa, se alcanza una dureza en el rango de los 700 HB [19]. 


\section{CONCLUSIONES}

El mayor contenido de molibdeno en la aleación A permitió la formación de una matriz austenítica, con bajas cantidades de perlita y bainita en estado de colada, pero además favoreció la estabilidad de la austenita retenida, obteniendo como resultado un leve aumento de dureza post tratamiento sub-cero, asociada a la formación de una baja cantidad de martensita.

Los tratamientos sub-cero aplicados a fundiciones blancas de matriz austenítica estabilizada con considerables cantidades de molibdeno (Aleación A), no presentan resultados óptimos para la fabricación de componentes donde se requieran considerables niveles de dureza, ya que se obtendrán importantes cantidades de austenita retenida post tratamiento.

Por su parte, la aleación B presentó una matriz formada por austenita retenida, colonias perlíticas y bainita, ubicadas mayoritariamente en el seno de la estructura dendrítica de colada, debido a su menor contenido de Mo esta aleación presentó una transformación de austenita retenida a martensita aparentemente completa $\mathrm{a}-180^{\circ} \mathrm{C}$.

\section{AGRADECIMIENTOS}

Los autores desean agradecer a la empresa ME Elecmetal y al Departamento de Ingeniería Metalúrgica de la Universidad de Santiago de Chile, por el apoyo al presente trabajo. S. Ordoñez agradece a la Universidad de Santiago de Chile por el apoyo a través del proyecto 1555LD.

\section{BIBLIOGRAFÍA}

[1] ASTM Standard A 532/A 532 M - 93a, "Standard Specification for Abrasion-Resistant Cast Irons", ASTM International, West Conshohocken, PA, DOI: 10.1520/A0532_A0532M-10R14, www.astm.org.

[2] ASM International, ASM Metals Handbook Volume 01 Properties and Selection Irons, Steels, and HighPerformance Alloys, 10 ed., Ohio, ASM International, 1990, pp. 150-157.

[3] LAIRD, G., GUNDLACH, R., RÖHRIG,K., Abrasion-Resistant Cast Iron Handbook, 1 ed., Illinois, American Foundry Society, 2000.

[4] CORTÉS, E., BEDOLLA, A., MEJÍA, I., et al., "Effects of tungsten on the microstructure and on the abrasive wear behavior of a high-chromium white iron", Wear, v. 376-377, pp. 77-86, Febrero 2017.

[5] GRUPTA, S., KHANDELWAL, A., GHOSE, A., et al., "Slurry erosion behavior of destabilized and deep cryogenically treated $\mathrm{Cr}-\mathrm{Mn}-\mathrm{Cu}$ white cast irons", Tribology in Industry, v. 38, n. 4, pp. 486-495, Enero 2016.

[6] WIENGMOON, A., Carbides in high chromium cast irons, Phitsanulok, Naresuan University, Department of Physics, Faculty of Science, Phitsanulok, 2011.

[7] EFEMENKO, V., CHABAK, Y., BRYKOV, M., "Kinetic parameters of secondary carbide precipitation in high-Cr white iron alloyed by Mn-Ni-Mo-V complex", Journal of Materials Engineering and Performance, v. 22, n. 5, pp. 1378-1385, Mayo 2013.

[8] DAVIS, R., Cast Irons. ASM Specialty Handbook, 1ed., Ohio, ASM International, ISBN: 978-0-87170564-8, 1996, pp. 107-123.

[9] HIGUERA, O., DUMITRU, F., MESA, D., "Improvement of abrasive wear resistance of the high chromium cast iron ASTM A-532 through thermal treatment cycles", Facultad de Ingeniería, v. 25, n. 41, pp. 93-103, Abril 2016.

[10] BEDOLLA, A., RAINFORTH, M., "Abrasive wear of V-Nb-Ti alloyed high-chromium white irons", Wear, v. 332-333, pp. 1006-1011, Junio 2015.

[11] ALBERTIN, E., SINATORA, A., PITALUGA, G., et al., "Relación entre factores micro- estructurales e impacto repetido en aleaciones de alto cromo para bolas de molino", Ingeniería Mecánica, v. 11, n. 1, pp. 5762, Enero 2008.

[12] MATSUBARA, Y., Research and development of abrasion wear resistant cast alloys for rolls of rolling and pulverizing mills, Kurume National College of Technology, Fukuoka, Japon.

[13] INTHIDECH, S., SRICHAROENCHAI, P., MATSUBARA, Y., "Effect of alloying elements on heat treatment behavior of hypoeutectic high chromium cast iron", Materials Transactions, v. 47, pp. 72-81, Enero 2006. 
[14] BEDOLlA, A., GUERRA, F., MEJÍA, I., et al., "Boron effect on the precipitation of secondary carbides during destabilization of a high-chromium white iron", In: Proceedings of the 10th International Symposium on the Science and Processing of Cast Iron, Mar del Plata, Argentina, Noviembre 2014.

[15] EFREMENKO, V., SHIMIZU, K., CHABAK, Y., "Effect of destabilizing heat treatment on solid-state phase transformation in high-chromium cast irons", METALLURGICAL AND MATERIALS TRANSACTIONS, v. 44, n. 12, pp. 5434-5446, Diciembre 2013.

[16] ASM International, ASM Metals Handbook Volume 04 Heat Treating, 10 ed., Ohio, ASM International, 1990, pp 481-490.

[17] ORLOWICZ, A., TUPAJ, M., MRÓZ, M., et al., "Abrasive wear resistance of a quenched and sub-zero treated high-chromium white cast iron", Metalurgija, v. 56, n. 3-4, pp. 304-306, Abril 2017.

[18] KUMAR, P., CHATTOPADHYAY, K., CHAKRABARTY, I., "Effect of sub-critical tempering and deep cryogenic treatment on slurry erosion of $\mathrm{Cr}-\mathrm{Mn}-\mathrm{Cu}$ white cast irons", ISIJ International, v. 54, n. 10, pp. 2294-2301, Mayo 2014.

[19] RANOD, R., Cryogenically super-hardened high-chomium white cast iron and method thereof, Patente: 5183518, USA, 2 de Febrero de 1993.

[20] MAALEKIAN, M., The Effects of Alloying Elements on Steels (I), 1 ed., Graz, Austria, Technische Universität Graz, 2007, pp. 15-16.

[21] VANDER, G., VAC AERO INTERNATIONAL INC., Martensite and the Control of Retained Austenite,

https://vacaero.com/information-resources/metallography-with-george-vander-voort/1391-martensite-andthe-control-of-retained-austenite.html. Accedido en Octubre de 2016.

[22] LINDE AG., Sub-zero Treatment of Steels,

http://www.pangas.ch/internet.lg.lg.che/de/images/Subzero_Treatment_of_Steels_en553_116014.pdf.

Accedido en Octubre de 2016.

[23] VIDYARTHI, M., GHOSE, A., CHAKRABARTY, I, "Effect of deep cryogenic treatment on the microstructure and wear performance of $\mathrm{Cr}-\mathrm{Mn}-\mathrm{Cu}$ white cast iron grinding media", Cryogenic, v. 58, pp. 85-92, Octubre 2013

[24] LIU, S., WU, X., SHI, L., et al., "Influence of cryogenic treatment on microstructure and properties improvement of die steel", Journal of Materials Science and Chemical Engineering, v. 3, pp. 37-46, Septiembre 2015.

[25] PATIL, P., TATED, R., "Comparison of effects of cryogenic treatment on different types of steels: A Revie", In: IJCA Proceedings on International Conference in Computational Intelligence (ICCIA2012), International Journal of Computer Applications, pp. 10-29, New York, USA, Marzo 2012.

[26] SU, Y., LI, D., ZHANG, X., "Optimizing hardenability of high chromium white cast iron", China Foundry, v. 3, n. 4, pp. 284-287, Noviembre 2006.

[27] IKEDA, M., UMEDA, T., TONG, C., et al., "Effect of molybdenum addition on solidification structure, mechanical properties and wear resistivity of high chromium cast irons", ISIJ International, v. 32, n. 11, pp. 1157-1162, 1992.

[28] GUERRA, F., BEDOLLA, A., MEJÍA, I., et al., "Precipitation of secondary carbides on high-chromium white cast irons with coopper aditions", In: Anales del XIX Congreso y Exposición Internacional de la Industria de la Fundición, Monterrey, México, Septiembre 2014.

[29] MARATRAY, F., POULALION, A., "Austenite retention in high-Cr white irons", AFS Transactions, v. 90, pp. 795-804, 1982. 